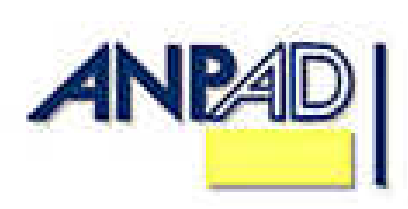

Disponível em http://www.anpad.org.br/rac

RAC, Curitiba, v. 13, n. 2, art. 1, p. 172-188, Abr./Jun. 2009

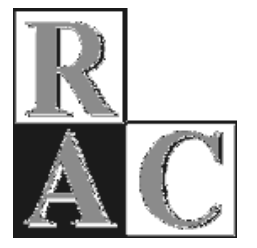

\title{
Indicadores e Características da Gestão de Fontes Externas de Informação Tecnológica e do Desempenho Inovador de Empresas Brasileiras
}

\section{Practices of the Management of External Sources of Technological Information and Innovative Performance of Brazilian Companies}

Clandia Maffini Gomes * Doutora em Administração pela USP. Professora do Departamento de Ciências Administrativas da UFSM, Santa Maria/RS, Brasil.

Isak Kruglianskas

Doutor pela USP.

Professor Titular e Chefe do Departamento de Administração da USP, São Paulo/SP, Brasil.

\footnotetext{
* Endereço: Clandia Maffini Gomes

Universidade Federal de Santa Maria, Rua Floriano Peixoto, 1184, Santa Maria/RS, 97015-700. E-mail: clandia@smail.ufsm.br
}

Copyright (C) 2009 RAC. Todos os direitos, inclusive de tradução, são reservados. É permitido citar parte de artigos sem autorização prévia desde que seja identificada a fonte. 


\section{RESUMO}

O estudo constitui parte de uma pesquisa maior, que procurou analisar a relação de algumas práticas de gestão de fontes externas da informação tecnológica com o desempenho inovador da empresa. No presente artigo são apresentadas as principais praticas para a gestão de fontes externas de informação tecnológica e os principais indicadores de desempenho inovador. A amostra foi composta por empresas do setor industrial que são associadas à ANPEI ou/e integrantes do cadastro do PGT/USP. A amostra contém 72 empresas (38\% do universo da pesquisa). Os resultados evidenciaram que a maioria das empresas é de grande porte e de setores tecnologicamente sofisticados. As principais modalidades de acesso às informações tecnológicas são: a aquisição por compra de tecnologia, a parceria com fornecedores, a aproximação com as universidades e a contratação de consultores. As principais fontes de informação são: Departamento de P\&D, Outros Departamentos, os Fornecedores, as Feiras e Exibições, as Universidades, Clientes, Network e os Institutos de Pesquisa. Os principais indicadores de desempenho inovador utilizados estão relacionados a produtos e/ou processos. Finalmente, a pesquisa sugere que o processo da gestão de fontes externas de informação para inovação tecnológica é pouco formalizado e carece de uma perspectiva estratégica.

Palavras-chave: gestão; fontes externas de informação; inovação tecnológica.

\section{ABSTRACT}

The study is part of a larger research project that sought to analyze the relationship between some management practices of external sources of technological information with the innovative performance of the enterprise. In this paper are presented the main practices for the management of external sources of technological information and some of the main indicators of innovation performance. The sample was composed of industrial enterprises associated with ANPEI and by companies on the mailing list of PGT/USP. The final sample had 72 subjects (38\% of the research universe). The results suggest that most of the companies in the sample are large and belong to technologically sophisticated industrial sectors. The main access modalities to technological information are performed by: technology acquisition, partnership with suppliers and with universities. The main sources of technological information are: the R\&D Department, Other Departments, Suppliers, Fairs and Exhibitions, Universities, Clients, Network and Research Institutes. The main innovation performance indicators used are closely related to products and/or processes. Finally, the results of the research suggest that the management of external sources of technological information for innovation lacks more formalization and strategic perspective.

Key words: management; external sources of information; technological innovation. 


\section{INTRODUÇÃO}

O estudo relatado neste artigo constitui parte de uma pesquisa mais ampla, que buscou entender a relação entre alguns elementos das práticas de gestão de fontes externas de informação tecnológica com alguns elementos do desempenho inovador da empresa. Neste artigo são apresentados os principais indicadores e características que foram identificados na pesquisa. Apesar da aceleração da inovação, a partir da utilização de fontes externas de informação tecnológica, poucas organizações possuem uma estratégia orientada para a gestão dessas fontes. As atividades efetuadas nesse sentido freqüentemente são realizadas de forma isolada.

A gestão de fontes de informação não ajuda somente à organização a combinar as fontes de informação internas e externas a serem consideradas, mas também a ampliar as suas atividades de inovação. As empresas estão incrementando as alianças para o uso de fontes externas de informação para inovação, porém ainda carecem de uma estratégia explícita de gestão.

A pesquisa survey foi realizada em empresas industriais brasileiras com características inovadoras. A análise de práticas de gestão em empresas de primeira linha serve de base para a disseminação do tema, ainda pouco explorado no meio acadêmico, assim como uma orientação para as empresas que buscam o gerenciamento e a integração das diversas fontes de informação para a inovação, visando à maximização dos seus resultados.

Os desafios impostos pelo ambiente globalizado, em que a capacidade de inovar assume caráter decisivo nas relações econômicas entre empresas e entre países, a gestão da inovação tecnológica transforma-se em vasto campo de atuação, especialmente para o Brasil, cuja base de desenvolvimento tecnológico ainda se encontra em construção.

\section{GESTÃo DE FONTES EXTERNAS DE INFORMAÇÃO PARA A INOVAÇÃO}

A elaboração de uma estratégia de inovação inclui a definição de objetivos, de domínios desejáveis e de produtos. Para tanto é necessário delinear as fontes internas e externas que serão controladas e os princípios chaves da gestão de fontes que guiarão as decisões, visando a assegurar que os objetivos sejam atendidos (Linder, Jarvenpaa, \& Davenport, 2003b). Somente uma gestão integrada do processo de inovação pode assegurar o sucesso e a competitividade das organizações, transformando idéias em novas categorias de produtos, garantindo novos espaços no mercado e novos modelos de negócio.

O interesse em absorver tecnologia externa está aumentando substancialmente na comunidade de gerência de $\mathrm{P} \& \mathrm{D}$. As empresas estão, cada vez mais, explorando formas de relacionamento do negócio com fontes externas de tecnologia. A maior dificuldade consiste em reconhecer o aspecto estratégico da gestão de fontes externas de informação para a inovação.

Diversos estudos foram realizados, tentando identificar as fontes de informação e seu relacionamento com a inovação organizacional. Hartman, Tower e Sebora (1994) afirmam que a importância e a frequiência de uso de fontes de idéias em empresas constituem assuntos de interesse crescente em pesquisas. Os gerentes são consumidores regulares de informação externa e de trocas verbais com pessoas da área de marketing. As fontes pessoais, escritas e informais são mais usadas.

Em um estudo desenvolvido junto ao setor de telecomunicações, Porto, Prado e Plonski (2003) afirmam que as fontes mais utilizadas, de acordo com os dados da pesquisa, são o departamento de P\&D da Matriz, o departamento de P\&D interno, as universidades, os institutos de pesquisa, bem como a participação em conferências, simpósios, feiras e exposições. Os institutos de pesquisa e as universidades são pouco utilizados e apresentam pouca freqüência de uso. As conferências, simpósios, feiras e exposições e as publicações especializadas também são pouco utilizadas. Os fornecedores, os 
clientes e as empresas de consultoria são fontes muito pouco utilizadas. A aquisição de patentes, licenças e know-how, outros departamentos da empresa, empresas dentro do grupo e concorrentes constituem fontes não utilizadas pela maioria das empresas pesquisadas.

Laursen e Salter (2004) analisaram as fontes de informação e de conhecimento para a inovação em empresas de manufatura inglesas. Os resultados indicaram que as fontes internas são as mais utilizadas, seguidas pelas fontes externas ligadas ao mercado. As atividades de inovação das empresas inglesas são determinadas pelas suas relações com fornecedores e consumidores e pelo modo como organizam suas atividades internas de suporte da inovação. As fontes de idéias para a inovação também foram identificadas na abordagem de Salter e Gann (2002). O estudo efetuado com funcionários de uma empresa inglesa evidenciou que as principais fontes de idéias utilizadas pelas empresas são internas e pessoais e são provenientes de conversas com colegas, projetos e experiências anteriores. Do mesmo modo, o estudo desenvolvido por Linder, Jarvenpaa e Davenport (2003a), evidenciou que a utilização de fontes externas de informação para a inovação tende a crescer substancialmente nos próximos anos. As organizações têm movido seu foco de fontes internas para fontes externas de informação para a inovação, a partir do envolvimento em venture capital, alianças ou aquisições de tecnologia. Apesar do uso de fontes externas de informação para a inovação, as empresas, de modo geral, não possuem uma estratégia de gestão dessas fontes.

As empresas podem olhar para outras empresas para inovar, mas elas raramente o fazem de forma sistemática e sob a perspectiva de uma estratégia explicita, freqüentemente limitando-se a uma única fonte, de forma isolada. Poucas empresas têm uma estratégia de gestão de fontes de informação para a inovação, gerenciando de forma integrada as diversas fontes para obter mais resultados. As empresas mais inovadoras gerenciam diversos conjuntos de canais de inovação, que incorporam não somente conjuntos de fontes, mas também irão estabelecer abordagens de gestão para as interfaces. Elas cultivam múltiplas fontes externas e explicitam uma clara estratégia de uso de fontes de informação para a inovação (Linder et al., 2003a).

Ao mesmo tempo, destacam Beltramo, Mason e Paul (2004), a capacidade de uma empresa expandir seu conhecimento a partir do uso de fontes externas de informação deriva da combinação de vários relacionamentos, que podem ser formais ou informais - envolvendo outras empresas, colaboração interfirmas (envolvendo consumidores e fornecedores) e a difusão de tecnologia entre empresas envolvendo departamentos de universidades ou laboratórios de setores públicos e privados, assim como da capacidade de network dos trabalhadores em pesquisa e desenvolvimento, construindo relacionamento individual com cientistas e engenheiros de outras empresas e organizações. Poucas companhias possuem um foco de inovação corporativo, raramente têm como meta um modelo de inovação e o processo de inovação do negócio tende a acontecer a partir de iniciativas ocasionais de reengenharia. Dentre as principais vantagens no uso de fontes externas, destacam-se: a criação de novas oportunidades, resultados mais rápidos e eficazes, diminuição dos custos da inovação, maior facilidade na definição de prioridades e estímulo à inovação interna.

Observa-se assim, a importância que a gestão das fontes de informação para a inovação apresenta no contexto atual das organizações. A gestão do processo de inovação, mais do que o desenvolvimento interno deve visar à captura de valor. Além disso, a mensuração do desempenho é essencial, ainda que de modo imperfeito.

\section{AVALIAÇÃO DO DESEMPENHO INOVADOR}

Com o objetivo de definir indicadores para a mensuração da eficácia da gestão de fontes externas de inovação nas empresas, foram identificadas na literatura algumas das principais medidas utilizadas pelas empresas, visando à avaliação do seu desempenho inovador. A disponibilidade das informações exerce papel importante nas decisões dos analistas que podem abandonar medidas com alta capacidade de prever valor, dado seu elevado custo de obtenção e a busca de uma análise de curto prazo 
decorrente da volatilidade da economia. Deve-se destacar a ampliação da influência das informações não-financeiras utilizadas pelos analistas e o maior interesse em fatores relacionados à estratégia, à credibilidade da administração, à inovação e ao posicionamento mercadológico (Low, 1998, como citado em Pace, Basso, \& Silva, 2003).

De acordo com Costa e Cunha (2001), a capacitação tecnológica pode ser medida por meio de diferentes indicadores, mas todos se referem à infra-estrutura, à capacitação dos recursos humanos envolvidos em $\mathrm{P} \& \mathrm{D}$, às fontes externas de aquisição de tecnologia e aos resultados alcançados. Na pesquisa efetuada junto aos setores de metalurgia, mecânica e eletroeletrônica foram identificados, como principais indicadores, a automação industrial, a capacidade de gerar tecnologia, o número de funcionários em $\mathrm{P} \& \mathrm{D}$, a importância atribuída ao ramo de $\mathrm{P} \& \mathrm{D}$ e o percentual de faturamento anual investido em $\mathrm{P} \& \mathrm{D}$.

Uma das principais dificuldades para a análise do comportamento inovador das empresas consiste na disponibilidade de dados. Para Sbragia, Kruglianskas e Andreassi (1998), os indicadores apresentados por diversos países são muito incipientes e limitados. Neste sentido, observa-se que várias instituições internacionais se têm empenhado em criar e definir indicadores comuns.

Dentre esses estudos podem-se destacar os dados apresentados pela Third Community Innovation Survey [CIS3] (Eurostat, 2004). A pesquisa apresenta dados relativos ao período de 1998 a 2000 em países da União Européia, Noruega e Islândia. A pesquisa analisa os efeitos e os impactos das atividades de inovação, a partir da análise de três dimensões fundamentais: a importância dos resultados da inovação em termos de faturamento, os efeitos da inovação no modo de a empresa conduzir o negócio e a proteção dos resultados da inovação (patentes e métodos de proteção similares). O faturamento não é por si só uma medida de sucesso na empresa. Um alto valor de faturamento não implica necessariamente alto desempenho. Porém, não obstante, constitui-se em uma mensuração de resultado. A medida de faturamento pode ser analisada em relação à atividade de inovação das empresas. Os principais efeitos da atividade de inovação, segundo os dados obtidos na pesquisa, são relacionados ao produto. A proporção de empresas com atividade de inovação que consideraram que sua atividade de inovação teve significativo impacto foi mais alta entre as empresas do setor industrial. Os dados evidenciaram, também, alta proporção de empresas com atividade de inovação que solicitaram o registro de pelo menos uma patente para proteger invenções ou inovações.

Torna-se insuficiente associar tecnologia e competitividade, pois não existe ligação direta entre tecnologia e competitividade. A passagem de uma para a outra é feita mediante a inovação, transformando o potencial (recursos tecnológicos) em bem econômico (produtos). Esse processo fundamental consiste em dominar tecnologias adaptadas, para apoiar a capacidade de inovar e realizar a inovação para construir a competitividade por meio de aplicações que correspondem às necessidades da clientela. Para passar à ação, afirmam Ribault, Martinet e Lebidois (1995), importa haver orientação estratégica para canalizar os esforços de inovação nas áreas de produtos e de serviços, bem como meios e competências para a concepção e o desenvolvimento. A finalidade das políticas que sustentam a estratégia da empresa consiste em contribuir para os objetivos de competitividade.

\section{METODOLOGIA}

A pesquisa, de natureza quantitativa, envolveu a realização de uma enquête (survey). De acordo com Babbie (1999), os estudos survey são realizados para permitir enunciados descritivos de uma população. Para tanto foi aplicado um instrumento de coleta de dados em empresas brasileiras, visando a identificar como a gestão de fontes externas de informação para a inovação influencia o desempenho inovador das empresas participantes da pesquisa. 


\section{Modelo Conceitual}

Tendo por base os objetivos do estudo, os conceitos e as informações obtidos na fundamentação teórica, assim como o conhecimento aprendido na fase exploratória da pesquisa, foi elaborado o modelo conceitual básico da pesquisa, formado por um conjunto de variáveis relacionadas à gestão de fontes externas de informação tecnológica e ao desempenho inovador. O modelo conceitual adotado é ilustrado pela Figura 1, apresentada a seguir.

\section{Figura 1: Modelo Conceitual da Pesquisa}

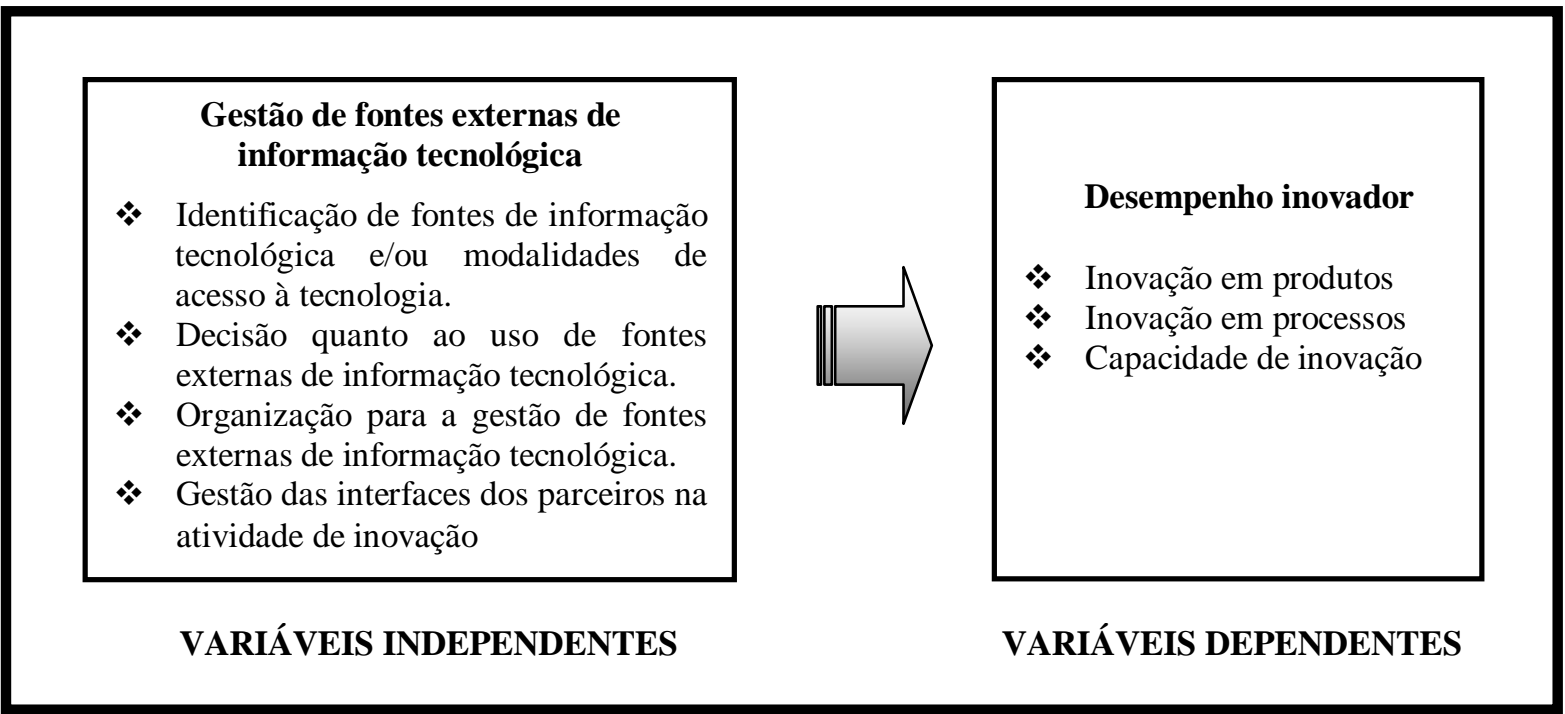

\section{Amostragem e Procedimentos para Coleta e Análise dos Dados}

\section{Composição da Amostra}

O universo da pesquisa foi constituído de empresas brasileiras do setor industrial com características e indícios de ênfase na atividade de inovação. A escolha dessa população como objeto de investigação prendeu-se ao fato de que é nesse tipo de empresa que ocorre, de forma mais enfática, a gestão de fontes externas de informação tecnológica. A base cadastral das empresas convidadas para participarem da pesquisa foi a relação de empresas ligadas à Associação Nacional de Pesquisa, Desenvolvimento e Engenharia das Empresas Inovadoras [ANPEI] e a relação das empresas que integram a mailing list do PGT Núcleo de Gestão Tecnológica da USP e do Programa de Gestão Tecnológica [PGT] da Fundação Instituto de Administração.

O cadastro de empresas disponível na base de dados da ANPEI conta com 75 empresas nacionais do setor industrial e de serviços relativas à atividade de inovação tecnológica. Em função dos objetivos da pesquisa, a base cadastral utilizada na pesquisa foi reduzida para 68 empresas, restringindo a amostra apenas às empresas com atividade industrial ou de transformação. Foram excluídas as empresas que atuam exclusivamente com a prestação de serviços de consultoria nas áreas de suporte tecnológico e de legislação.

O cadastro de empresas do PGT era composto por 137 empresas. Após o cruzamento de ambas as bases cadastrais, foram excluídas 14 empresas, por já constarem da base da ANPEI. Dessa forma o universo das empresas pesquisadas foi composto por 191 empresas: 68 (36\%) empresas ligadas à ANPEI e 123 (64\%) ligadas ao PGT. 
Portanto cabe ressaltar que a amostragem foi intencional e oportunista; assim, os resultados não podem ser generalizados para outras empresas além daquelas que integraram a amostra utilizada. Todavia, dado o fato de as empresas estudadas serem empresas que demonstram alto interesse pela inovação tecnológica, os resultados obtidos podem ser bastante sugestivos sobre o que ocorre nas empresas inovadoras brasileiras.

\section{Procedimentos para Coleta e Análise dos Dados}

O questionário foi direcionado aos responsáveis pela área de tecnologia, diretores ou presidentes das empresas. A forma de envio utilizada foi por meio de e-mail e de acesso ao web site. A coleta de dados foi efetuada durante 75 dias (de outubro a dezembro de 2006). Nesse período, além do contato via email, foi utilizado o contato telefônico com as empresas, objetivando obter o maior número possível de respostas. O total de respostas obtidas foi elevado, considerando-se a base cadastral utilizada. De um total de 191 empresas foram recebidos 72 questionários, cerca de 38\%. Um índice de resposta acima de $25 \%$ é considerado elevado pela maioria dos especialistas. Devem-se considerar ainda as dificuldades em obter informações com um grupo de empresas, com destaque na área de inovação tecnológica, em que as questões de secret agreements são bastante comuns. Somam-se a isso as implicações e a existência de questões relativas ao desempenho das empresas, informações muitas vezes delicadas em setores com alto grau de competitividade.

Os dados foram analisados com auxílio dos softwares Excel e SPSS. A análise univariada dos dados obtidos foi efetuada a partir da verificação das medidas de tendência central, que permitem identificar as principais freqüências observadas em relação à determinada variável.

\section{ANÁLISE DOS RESULTADOS}

Com base nos dados obtidos, passa-se, agora, à análise, inicialmente das características que constituem o perfil dos respondentes e das empresas que compõem a amostra. Na seqüência são apresentados os valores assumidos pelas variáveis relativas à gestão de fontes externas de informação tecnológica e pelas variáveis relativas ao desempenho inovador.

\section{Perfil da Amostra}

O perfil predominante das empresas pesquisadas é apresentado na Tabela 1 a seguir.

Tabela 1: Perfil das Empresas

\begin{tabular}{|l|l|}
\hline Setor de atividade & $\begin{array}{l}\text { Material eletrônico e telecomunicações, química, } \\
\text { veículos/autopeças }\end{array}$ \\
\hline Número de empregados & Empresas de grande porte (acima de 500 funcionários) \\
\hline Receita operacional bruta & Superior a 400.000.000 reais \\
\hline Origem do capital controlador & Nacional \\
\hline Participação do capital estrangeiro & $\begin{array}{l}\text { Dentre as empresas que possuem capital estrangeiro, a } \\
\text { participação é superior a 50\%. }\end{array}$ \\
\hline Nacionalidade do capital estrangeiro & Estados Unidos, Europa e Ásia \\
\hline $\begin{array}{l}\text { Participação das exportações na receita } \\
\text { operacional bruta }\end{array}$ & $\begin{array}{l}\text { A maioria das empresas não possui atividade de exportação e, } \\
\text { entre as que exportam, o índice é de até 10\% }\end{array}$ \\
\hline Tipo de inovação pela enjunta & $\begin{array}{l}\text { Anovação em produto e em processo de forma conjunta em cooperação com outras empresas e/ou institutos e } \\
\text { universidades. }\end{array}$ \\
\hline $\begin{array}{l}\text { Responsabilidade principal } \\
\text { atividade de inovação }\end{array}$ & Diretoria ou gerência de P\&D \\
\hline $\begin{array}{l}\text { Área (setor) responsável principal pela } \\
\text { gestão das atividades de inovação }\end{array}$ &
\end{tabular}


A maioria das empresas caracteriza-se como de grande porte (número de empregados e receita) e concentra-se em setores de maior sofisticação tecnológica. O grupo de empresas apresenta, portanto, perfil atraente para a identificação das características da gestão de fontes externas de informação tecnológica a que o estudo se propõe.

\section{Gestão de Fontes Externas de Informação Tecnológica}

A gestão de fontes externas de informação tecnológica é caracterizada a partir da avaliação da intensidade de uso em relação ao seguinte: tipos de modalidades de acesso à tecnologia; tipos de fontes de informação tecnológica; critérios que orientam a escolha de fontes externas de informação tecnológica; fatores que dificultam o processo de contratação de fontes externas de informação tecnológica; o relacionamento com os parceiros na gestão de projetos; e benefícios da parceria/colaboração na atividade de inovação.

\section{Tipos de Modalidades de Acesso à Tecnologia}

A análise da intensidade de uso dos vários tipos de modalidades de acesso à tecnologia é efetuada a partir da interpretação dos dados da Tabela 2.

Tabela 2: Tipos de Modalidades de Acesso à Tecnologia

\begin{tabular}{|l|c|c|c|c|c|c|c|}
\hline \multirow{2}{*}{ Modalidades de Acesso } & \multicolumn{7}{|c|}{ Intensidade (\%) } \\
\cline { 2 - 9 } & NR & MBaixa & Baixa & Média & Alta & MAlta & Total \\
\hline Compras por especificação & 15,3 & 6,9 & 9,7 & 20,8 & 23,6 & 23,6 & 100 \\
\hline Parceria com fornecedores & 15,3 & 5,6 & 15,3 & 23,6 & 29,2 & 11,1 & 100 \\
\hline Universidades & 8,3 & 11,1 & 8,3 & 27,8 & 29,2 & 15,3 & 100 \\
\hline Contratação de consultoria & 9,7 & 18,1 & 16,7 & 25 & 26,4 & 4,2 & 100 \\
\hline Parceria com outras empresas & 16,7 & 16,7 & 11,1 & 22,2 & 22,2 & 11,1 & 100 \\
\hline Fóruns de interesse especial & 23,6 & 16,7 & 19,4 & 15,3 & 20,8 & 4,2 & 100 \\
\hline Subcontratação & 25,0 & 22,2 & 13,9 & 22,2 & 11,1 & 5,6 & 100 \\
\hline Compras por catálogo & 23,6 & 40,3 & 16,7 & 11,1 & 6,9 & 1,4 & 100 \\
\hline Parceria com concorrentes & 37,5 & 37,5 & 20,8 & 4,2 & - & - & 100 \\
\hline Aquisição de licença & 31,9 & 34,7 & 18,1 & 9,7 & - & 5,6 & 100 \\
\hline Venture capital & 56,9 & 30,6 & 8,3 & 2,8 & - & 1,4 & 100 \\
\hline Investimento capital de risco & 51,4 & 30,6 & 8,3 & 5,6 & 2,8 & 1,4 & 100 \\
\hline Consórcio de empresas & 37,5 & 29,2 & 12,5 & 12,5 & 6,9 & 1,4 & 100 \\
\hline Licenciamento & 31,9 & 27,8 & 6,9 & 16,7 & 11,1 & 5,6 & 100 \\
\hline Joint venture & 51,4 & 27,8 & 8,3 & 5,6 & 4,2 & 2,8 & 100 \\
\hline Aquisição de patentes & 44,4 & 27,8 & 18,1 & 8,3 & - & 1,4 & 100 \\
\hline Aquisição de empresas & 36,1 & 23,6 & 15,3 & 18,1 & 5,6 & 1,4 & 100 \\
\hline P\&D subcontratado & 33,3 & 23,5 & 11,1 & 13,9 & 12,5 & 5,6 & 100 \\
\hline Redes de cooperação & 29,2 & 20,8 & 12,5 & 18,1 & 9,7 & 9,7 & 100 \\
\hline Aliançaa estratégica & 36,1 & 19,4 & 15,3 & 16,7 & 5,6 & 6,9 & 100 \\
\hline
\end{tabular}

A compra por especificação constitui-se na modalidade de acesso à tecnologia com maior intensidade de uso por parte das empresas em função da possibilidade de adequação da tecnologia aos padrões previamente estabelecidos. As parcerias com outras empresas e com fornecedores, as universidades, a contratação de consultoria e os fóruns de interesse especial também são modalidades bastante utilizadas. Observa-se uma busca crescente por acesso à tecnologia a partir da associação e da parceira com outras instituições. Essa percepção corrobora os resultados de outras pesquisas realizadas 
sobre o tema que apontam a existência de uma tendência de crescimento na obtenção de tecnologia, a partir do relacionamento externo.

Os dados sugerem menor utilização de modalidades de acesso à tecnologia, a partir de terceiros, tais como compras por catálogo, subcontratação e licenciamento. A parceria com concorrentes, o consórcio de empresas e as redes de cooperação também são formas de acesso à tecnologia pouco valorizadas, dado que refletem traços da cultura empresarial predominante que não identifica as vantagens da atividade colaborativa. O investimento e a compra de tecnologia também não constituem práticas correntes, em razão da pouca importância atribuída ao investimento em venture capital, em joint venture, ao investimento em capital de risco, à aquisição de empresas, de patentes e de licenças, o que pode indicar a existência de pouco conhecimento acerca das vantagens de uso desse tipo de modalidade, assim como devido aos riscos e dificuldades culturais, legais, burocráticas e financeiras envolvidos.

\section{Tipos de Fontes de Informação Tecnológica}

O comportamento das empresas em relação aos tipos de fontes de informação tecnológica utilizadas pelas empresas pesquisadas é apresentado na Tabela 3.

Tabela 3: Tipos de Fontes de Informação Tecnológica

\begin{tabular}{|c|c|c|c|c|c|c|c|}
\hline \multirow[t]{2}{*}{ Fontes de informação } & \multicolumn{7}{|c|}{ Intensidade $(\%)$} \\
\hline & NR & MBaixa & Baixa & Média & Alta & MAlta & Total \\
\hline Departamento de P\&D & 5,6 & 2,8 & 8,3 & 6,9 & 22,2 & 54,2 & 100 \\
\hline Outros departamentos & 6,9 & 5,6 & 4,2 & 26,4 & 44,4 & 12,5 & 100 \\
\hline Fornecedores & 9,7 & 5,6 & 12,5 & 16,7 & 40,3 & 15,3 & 100 \\
\hline Feiras e exibições & 1,4 & 4,2 & 12,5 & 27,8 & 37,5 & 16,7 & 100 \\
\hline Universidades/centros educ. sup. & 4,2 & 6,9 & 13,9 & 22,2 & 33,3 & 19,4 & 100 \\
\hline Adoção de padrões tecnológicos & 13,9 & 12,5 & 11,1 & 18,1 & 30,6 & 13,9 & 100 \\
\hline Publicações técnicas e científicas & 1,4 & 5,6 & 15,3 & 27,8 & 29,2 & 20,8 & 100 \\
\hline Clientes & 6,9 & 5,6 & 19,4 & 20,8 & 27,8 & 19,4 & 100 \\
\hline Network & 9,7 & 12,5 & 13,9 & 22,2 & 25 & 16,7 & 100 \\
\hline Institutos de pesquisa & 8,3 & 9,7 & 23,6 & 13,9 & 23,6 & 20,8 & 100 \\
\hline Visitas a outras empresas/ licenc. & 13,9 & 19,4 & 11,1 & 31,9 & 15,3 & 8,3 & 100 \\
\hline Conf. científicas/profissionais & 4,2 & 8,3 & 11,1 & 29,2 & 26,4 & 20,8 & 100 \\
\hline Assoc.científicas/profissionais & 8,3 & 9,7 & 23,6 & 25 & 23,6 & 9,7 & 100 \\
\hline Redes inf. (on-line databases) & 8,3 & 12,5 & 19,4 & 25 & 20,8 & 13,9 & 100 \\
\hline Consumidores & 20,8 & 16,7 & 16,7 & 20,8 & 11,1 & 13,9 & 100 \\
\hline Visitas a outras emp. do grupo & 26,4 & 13,9 & 12,5 & 15,3 & 13,9 & 18,1 & 100 \\
\hline Contratação de talentos externos & 13,9 & 19,4 & 31,9 & 22,2 & 9,7 & 2,8 & 100 \\
\hline Empresas de consultoria & 8,3 & 11,1 & 29,2 & 27,8 & 15,3 & 8,3 & 100 \\
\hline Concorrentes & 15,3 & 8,3 & 27,8 & 18,1 & 25 & 5,6 & 100 \\
\hline Inst. testes, ensaios e certific. & 11,1 & 6,9 & 26,4 & 25 & 20,8 & 9,7 & 100 \\
\hline Comunidade de práticas & 40,3 & 22,2 & 26,4 & 5,6 & 2,8 & 2,8 & 100 \\
\hline Outras empresas do grupo & 30,6 & 9,7 & 19,4 & 12,5 & 16,7 & 11,1 & 100 \\
\hline P\&D de outras empresas & 20,8 & 29,2 & 20,8 & 18,1 & 9,7 & 1,4 & 100 \\
\hline Centros capacitação profissional & 13,9 & 29,2 & 27,8 & 19,4 & 8,3 & 1,4 & 100 \\
\hline Empresas contrat/ terceirizadas & 22,2 & 27,8 & 20,8 & 19,4 & 4,2 & 5,6 & 100 \\
\hline Redes comunitárias & 40,3 & 27,8 & 25 & 4,2 & 1,4 & 1,4 & 100 \\
\hline Aquis. lic., patentes, know-how & 29,2 & 25 & 19,4 & 11,1 & 11,1 & 4,2 & 100 \\
\hline Usuários líderes & 45,8 & 23,6 & 11,1 & 6,9 & 8,3 & 4,2 & 100 \\
\hline Outras & 86,1 & 5,6 & 2,8 & 1,4 & 1,4 & 2,8 & 100 \\
\hline
\end{tabular}

As fontes internas de informação mais utilizadas pelas empresas são, com maior destaque, os Departamentos de P\&D, seguido de outros departamentos da empresa. A seguir, destacam-se pela alta intensidade de uso os fornecedores, as feiras e as exibições, as universidades e outros centros de 
educação superior, a adoção de padrões tecnológicos, de saúde, segurança e ambientais em relação a consumidores e fornecedores, publicações técnicas e científicas, os clientes, network e institutos de pesquisa. Os dados obtidos corroboram as conclusões de vários pesquisadores que afirmam que as fontes de informação tecnológica mais utilizadas pelas empresas são as fontes de origem interna, havendo, entretanto, uma tendência ao incremento no uso de fontes externas. Em um estudo realizado com empresas brasileiras, Porto et al. (2003) afirmam que, dentre os principais tipos de fontes de informação para a inovação utilizados pelas empresas, encontra-se o departamento de P\&D da matriz, o departamento de P\&D interno, as universidades, os institutos de pesquisa, as conferências, os seminários, as feiras e as exposições.

As fontes decorrentes de relacionamentos com a comunidade externa apresentam média intensidade de uso, como no seguinte elenco: visitas a outras empresas do grupo e a outras empresas ou licenciadoras, participação em conferências e associações científicas e profissionais, associações científicas e profissionais, redes informatizadas (on-line databases), consumidores e visitas a outras empresas do grupo. Esse resultado evidencia a relativa importância que as empresas atribuem à participação em eventos de natureza técnico-científica, participação em associações, pesquisa em publicações científicas e visitas técnicas. Observa-se a convergência entre esses resultados e as conclusões do estudo de Fleury e Fleury (1997), ao evidenciar que publicações e visitas de natureza técnica estão entre as principais fontes de informação utilizadas pelas empresas brasileiras.

O P\&D de outras empresas, as empresas contratadas ou terceirizadas, os usuários líderes, as redes comunitárias e a aquisição de licenças, patentes e know-how são fontes de informação pouco utilizadas pelas empresas. Esses dados se alinham com as conclusões da Tabela 2, as quais evidenciam que as empresas atribuem pouca importância à tecnologia obtida a partir de instituições terceirizadas. Além disso, as informações sugerem que, assim como na questão anterior, as empresas possuem pouca experiência acerca da aquisição de tecnologia e de informações provenientes de licenças e de patentes, devido às dificuldades e aos riscos inerentes a esse tipo de prática. Os usuários líderes e as redes comunitárias são fontes bastante específicas e ainda pouco conhecidas e experimentadas pela maioria das empresas.

Os resultados obtidos confirmam a tendência da maior utilização por parte das empresas pesquisadas de fontes internas, apresentando, ao mesmo tempo, uma tendência de aumento no uso de fontes externas de informação tecnológica.

\section{Critérios que Orientam a Escolha de Fontes Externas de Informação Tecnológica}

Os dados relativos aos critérios que orientam a escolha das fontes externas de informação tecnológica das empresas participantes da pesquisa são apresentados na Tabela 4.

Tabela 4: Critérios que Orientam a Escolha de Fontes Externas de Informação Tecnológica

\begin{tabular}{|l|c|c|c|c|c|c|c|}
\hline \multirow{2}{*}{ Critérios } & \multicolumn{7}{|c|}{ Intensidade (\%) } \\
\cline { 2 - 9 } & NR & MBaixa & Baixa & Média & Alta & MAlta & Total \\
\hline Expertise & 9,7 & - & - & 4,2 & 34,7 & 51,4 & 100 \\
\hline Reputação/imagem & 6,9 & 4,2 & 2,8 & 13,9 & 51,4 & 20,8 & 100 \\
\hline Performance & 5,6 & - & 1,4 & 19,4 & 44,4 & 29,2 & 100 \\
\hline Custo & 4,2 & 1,4 & 5,6 & 36,1 & 41,7 & 11,1 & 100 \\
\hline Prazo & 9,7 & 5,6 & 5,6 & 27,8 & 41,7 & 9,7 & 100 \\
\hline Risco & 8,3 & 5,6 & 8,3 & 34,7 & 36,1 & 6,9 & 100 \\
\hline Oportunidade & 6,9 & 1,4 & 4,2 & 31,9 & 36,1 & 19,4 & 100 \\
\hline Flexibilidade em serviços & 8,3 & 2,8 & 16,7 & 33,3 & 27,8 & 11,1 & 100 \\
\hline Localização & 13,9 & 16,7 & 27,8 & 29,2 & 9,7 & 2,8 & 100 \\
\hline Outros & 90,3 & 2,8 & 1,4 & 2,8 & 1,4 & 1,4 & 100 \\
\hline
\end{tabular}


A expertise constitui-se no critério principal que influencia a escolha das fontes externas de informação tecnológica. Esse resultado confirma a importância estratégica do conhecimento e da especialização técnica na geração de valor aos produtos e aos processos para as empresas inovadoras.

A reputação e a performance são outros aspectos que influenciam a tomada da decisão. A credibilidade da fonte de informação tecnológica traz segurança em relação aos aspectos legais envolvidos, e a avaliação do desempenho garante a continuidade ou não dos contratos e das parcerias. A localização é fator pouco considerado pelas empresas, em razão da alta valorização da expertise.

Além da contribuição tecnológica, aspectos estratégicos como o custo, o risco e o prazo também definem a seleção de determinado tipo de fonte de informação tecnológica. Esse comportamento não poderia ser diferente, na medida em que realizar parcerias com fontes externas de informação altamente especializadas envolve investimentos de grande porte e a avaliação do risco envolvido torna-se ingrediente fundamental. A oportunidade de acesso e de obtenção da informação é outro aspecto considerado, o que ressalta a importância de possuir uma eficiente estrutura de network e da adoção de instrumentos de vigilância tecnológica, visando a identificar e captar essas oportunidades.

Ribault et al. (1995) dividem os critérios de escolha em estratégicos e táticos, os quais devem ser estabelecidos pela empresa a partir da estratégia tecnológica adotada. Com base nesse critério, pode-se dizer que os principais critérios adotados pelas empresas pesquisadas são de origem estratégica.

Para Leonard-Barton (1995), os principais aspectos que orientam as empresas no processo de aquisição de tecnologia são o acesso ao potencial tecnológico, a avaliação da expertise da fonte e a localização das empresas. Os resultados da pesquisa corroboram essa avaliação no que se refere à expertise da fonte, critério muito utilizado; porém divergem no critério de localização, pouco utilizado pelas empresas investigadas.

\section{Fatores que Dificultam o Processo de Contratação de Fontes Externas de Informação Tecnológica}

A avaliação dos principais aspectos que dificultam o processo de contratação de fontes externas de informação tecnológica é apresentada na Tabela 5.

Tabela 5: Fatores que Dificultam o Processo de Contratação de Fontes Externas de Informação Tecnológica

\begin{tabular}{|l|c|c|c|c|c|c|c|}
\hline Dificuldades no processo de & \multicolumn{7}{|c|}{ Intensidade (\%) } \\
\cline { 2 - 9 } contratação & NR & MBaixa & Baixa & Média & Alta & MAlta & Total \\
\hline Problemas de coordenação do processo & 9,7 & 9,7 & 22,2 & 13,9 & 34,7 & 9,7 & 100 \\
\hline Falta de pessoal especializado & 12,5 & 6,9 & 15,3 & 13,9 & 33,3 & 18,1 & 100 \\
\hline $\begin{array}{l}\text { Excesso de burocracia na formalização } \\
\text { das parcerias }\end{array}$ & 6,9 & 6,9 & 12,5 & 23,6 & 26,4 & 23,6 & 100 \\
\hline $\begin{array}{l}\text { Dificuldades relativas à proteção de } \\
\text { direitos autorais, marcas e patentes }\end{array}$ & 13,9 & 8,3 & 18,1 & 19,4 & 26,4 & 13,9 & 100 \\
\hline $\begin{array}{l}\text { Falta de comunicação entre os } \\
\text { parceiros }\end{array}$ & 13,9 & 9,7 & 19,4 & 20,8 & 25 & 11,1 & 100 \\
\hline $\begin{array}{l}\text { Dificuldades na efetivação de acordos } \\
\text { de licenciamento de tecnologia }\end{array}$ & 16,7 & 8,3 & 19,4 & 33,3 & 15,3 & 6,9 & 100 \\
\hline $\begin{array}{l}\text { Dificuldades relativas à legislação, } \\
\text { normas e padrões }\end{array}$ & 11,1 & 11,1 & 20,8 & 29,2 & 19,4 & 8,3 & 100 \\
\hline Alto custo do processo de contratação & 9,7 & 8,3 & 23,6 & 27,8 & 16,7 & 13,9 & 100 \\
\hline Falta de habilidade de negociação & 12,5 & 19,4 & 20,8 & 18,1 & 18,1 & 11,1 & 100 \\
\hline Outros & 90,3 & 4,2 & 1,4 & 2,8 & 1,4 & 9,7 & 100 \\
\hline
\end{tabular}


Os aspectos relacionados a problemas de coordenação do processo, a falta de pessoal especializado, o excesso de burocracia na formalização das parcerias, aspectos relativos à proteção de direitos autorais, marcas e patentes e a falta de comunicação entre os parceiros constituem as principais dificuldades encontradas pelas empresas no processo de contratação de fontes externas de informação tecnológica. As dificuldades na efetivação de acordos de licenciamento e referentes à legislação, normas e padrões são consideradas aspectos secundários. A habilidade de negociação com os parceiros é considerada pouco importante para as empresas.

Os dados evidenciam que os principais limitadores do processo de contratação de fontes externas de informação tecnológica são estruturais, de relacionamento entre os parceiros e de origem legal. Isso se explica, fundamentalmente, pela falta de conhecimento, de especialização e de estrutura específicos para enfrentar os novos desafios relacionados à busca de informações e de tecnologia externos à empresa. Essa tendência, ao mesmo tempo que gera novas oportunidades de inovação, cria novos condicionantes estruturais, legais e culturais, seja no estabelecimento de regras para o gerenciamento das relações de parceria e de colaboração, seja no design dos arranjos legais que norteiam o processo de gestão.

\section{Relacionamento com os Parceiros na Gestão de Projetos}

Os dados relativos aos principais aspectos que facilitam e dificultam o relacionamento com os parceiros na gestão de projetos são apresentados na Tabela 6 .

Tabela 6: Relacionamento com os Parceiros na Gestão de Projetos

\begin{tabular}{|c|c|c|c|c|c|c|c|}
\hline \multirow{2}{*}{ Relacionamento com os parceiros } & \multicolumn{7}{|c|}{ Intensidade (\%) } \\
\hline & NR & MBaixa & Baixa & Média & Alta & MAlta & Total \\
\hline \multicolumn{8}{|l|}{ Facilidades no relacionamento } \\
\hline $\begin{array}{l}\text { Troca de informações entre os } \\
\text { parceiros }\end{array}$ & 2,8 & - & - & 33,3 & 47,2 & 16,7 & 100 \\
\hline $\begin{array}{l}\text { Nível de tecnologia semelhante entre } \\
\text { os parceiros }\end{array}$ & 8,3 & - & 8,3 & 20,8 & 45,8 & 16,7 & 100 \\
\hline $\begin{array}{l}\text { Existência de relacionamento anterior } \\
\text { entre os parceiros }\end{array}$ & 2,8 & - & 5,6 & 16,7 & 43,1 & 31,9 & 100 \\
\hline Expertise dos parceiros & 5,6 & 1,4 & 14 & 6,9 & 41,7 & 43,1 & 100 \\
\hline Controle de desempenho das parcerias & 5,6 & 6,9 & 8,3 & 30,6 & 38,9 & 9,7 & 100 \\
\hline Proximidade geográfica & 5,6 & 6,9 & 23,6 & 27,8 & 30,6 & 5,6 & 100 \\
\hline Outros & 91,7 & 4,2 & - & - & 2,8 & 1,4 & 100 \\
\hline \multicolumn{8}{|l|}{ Dificuldades no relacionamento } \\
\hline $\begin{array}{l}\text { Ritmo de trabalho diferenciado entre os } \\
\text { parceiros }\end{array}$ & 9,7 & 4,2 & 16,7 & 18,1 & 34,7 & 16,7 & 100 \\
\hline Falta de coordenação das atividades & 8,3 & 6,9 & 26,4 & 15,3 & 29,2 & 13,9 & 100 \\
\hline Estrutura organizacional pouco flexível & 9,7 & 11,1 & 19,4 & 18,1 & 27,8 & 13,9 & 100 \\
\hline $\begin{array}{lccc}\begin{array}{l}\text { Falta de pessoal } \\
\text { efetuar as atividades }\end{array} & & \\
\end{array}$ & 8,3 & 6,9 & 20,8 & 16,7 & 26,4 & 20,8 & 100 \\
\hline $\begin{array}{l}\text { Transferência dos elementos da cultura } \\
\text { do negócio }\end{array}$ & 11,1 & 6,9 & 27,8 & 27,8 & 16,7 & 9,7 & 100 \\
\hline Temor de perda de conhecimento & 8,3 & 16,7 & 29,2 & 22,2 & 11,1 & 12,5 & 100 \\
\hline Outros & 88,9 & 2,8 & 1,4 & 1,4 & 5,6 & 11,1 & 100 \\
\hline
\end{tabular}

No que se refere aos fatores que facilitam o relacionamento com os parceiros, observa-se que todos os aspectos avaliados são considerados importantes para a gestão do relacionamento com os parceiros. A troca de informações e o nível de tecnologia semelhante constituem elementos centrais no sucesso do estabelecimento de uma parceria em razão das necessidades de estabelecimento de uma linguagem comum e de manutenção de padrões e de normas técnicas entre os parceiros. A existência de relacionamento anterior entre os parceiros e a proximidade geográfica influencia a confiança e a 
comunicação entre os parceiros. A existência de mecanismos de controle do desempenho das parcerias possibilita a identificação do grau de contribuição e de responsabilidade de cada um, o que fortalece as relações existentes.

No que se refere aos fatores que dificultam o relacionamento com os parceiros observa-se que o ritmo de trabalho diferenciado entre os parceiros, a falta de coordenação das atividades, a estrutura organizacional pouco flexível, a falta de pessoal qualificado para efetuar as atividades representam os principais aspectos que dificultam o relacionamento com os parceiros. As dificuldades relacionadas à transferência dos elementos da cultura do negócio e do temor de perda de conhecimento pouco influenciam as relações estabelecidas com os parceiros.

Os resultados verificados levam ao entendimento de que os fatores que dificultam a gestão das parcerias são de origem interna, relativos à infra-estrutura de trabalho e à interação com os parceiros. Esses dados corroboram a análise efetuada a partir dos dados da Tabela 5, quanto aos fatores críticos para a contratação de fontes externas de informação tecnológica. Fica evidenciada a pouca estrutura e preparo das empresas na gestão, devido ao deslocamento do foco nas informações internas para as informações externas à empresa, onde a gestão do relacionamento entre os parceiros se torna um condicionante de sucesso da atividade de inovação.

\section{Benefícios da Parceria/Colaboração na Atividade de Inovação}

A avaliação dos benefícios da parceria e da colaboração na atividade de inovação é apresentada na Tabela 7.

Tabela 7: Benefícios da Parceria/Colaboração na Atividade de Inovação

\begin{tabular}{|l|c|c|c|c|c|c|c|}
\hline \multirow{2}{*}{ Benefícios da parceria e da colaboração } & \multicolumn{6}{|c|}{ Intensidade (\%) } \\
\cline { 2 - 8 } & NR & MBaixa & Baixa & Média & Alta & MAlta & Total \\
\hline Melhoria da imagem & 9,7 & 1,4 & 18,1 & 16,7 & 43,1 & 11,1 & 100 \\
\hline $\begin{array}{l}\text { Otimização do desenvolvimento do } \\
\text { produto com menor risco }\end{array}$ & 9,7 & 2,8 & 11,1 & 19,4 & 43,1 & 13,9 & 100 \\
\hline $\begin{array}{l}\text { Desenvolvimento e absorção de } \\
\text { tecnologia mais eficaz }\end{array}$ & 6,9 & 1,4 & 6,9 & 26,4 & 41,7 & 16,7 & 100 \\
\hline $\begin{array}{l}\text { Uso em parceria das instalações de } \\
\text { pesquisa e de informações tecnológicas }\end{array}$ & 6,9 & 5,6 & 11,1 & 27,8 & 37,5 & 11,1 & 100 \\
\hline $\begin{array}{l}\text { Acesso a recursos financeiros e a } \\
\text { recursos humanos qualificados }\end{array}$ & 6,9 & 2,8 & 6,9 & 33,3 & 36,1 & 13,9 & 100 \\
\hline Melhoria do potencial mercadológico & 8,3 & 2,8 & 11,1 & 26,4 & 34,7 & 16,7 & 100 \\
\hline Outros & 97,2 & 1,4 & - & 1,4 & - & - & 100 \\
\hline
\end{tabular}

Todos os benefícios da parceria/colaboração na atividade de inovação avaliados são considerados importantes pelas empresas pesquisadas. Em uma economia global, bastante sensível à velocidade das mudanças tecnológicas e à escassez de recursos, a atividade colaborativa assume caráter cada vez mais central e decisivo na atividade de inovação. Essa tendência pode ser visualizada nos dados obtidos, à medida que as empresas parecem buscar a realização de parcerias, tendo em vista, especialmente, a melhoria da imagem, a otimização do desenvolvimento de produto com menor risco e a absorção eficaz de tecnologia.

\section{Desempenho Inovador}

Os resultados da atividade de inovação são mensurados por meio da avaliação da intensidade das contribuições da atividade de inovação, da evolução dos indicadores nos últimos cinco anos e dos valores dos indicadores obtidos em 2005. 


\section{Contribuições da Atividade de Inovação}

Os dados relacionados aos efeitos da atividade de inovação são apresentados na Tabela 8 relacionada a seguir.

Tabela 8: Contribuições da Atividade de Inovação

\begin{tabular}{|l|c|c|c|c|c|c|c|}
\hline \multicolumn{1}{|c|}{ Contribuições } & \multicolumn{5}{c|}{ Intensidade (\%) } \\
\cline { 2 - 8 } & NR & MBaixa & Baixa & Média & Alta & MAlta & Total \\
\hline $\begin{array}{l}\text { Ampliação da gama de produtos } \\
\text { ofertados }\end{array}$ & 9,7 & 1,4 & 9,7 & 16,7 & 30,6 & 31,9 & 100 \\
\hline Melhoria da qualidade dos produtos & 8,3 & - & 4,2 & 12,5 & 47,2 & 27,8 & 100 \\
\hline Aumento da capacidade de produção & 11,1 & - & 13,9 & 20,8 & 41,7 & 12,5 & 100 \\
\hline Melhoria da flexibilidade de produção & 11,1 & - & 8,3 & 29,2 & 40,3 & 11,1 & 100 \\
\hline Entrada em novos mercados & 13,9 & 1,4 & 2,8 & 16,7 & 37,5 & 27,8 & 100 \\
\hline $\begin{array}{l}\text { Ampliação da participação da empresa } \\
\text { no mercado }\end{array}$ & 12,5 & - & 5,6 & 18,1 & 36,1 & 27,8 & 100 \\
\hline Redução dos custos de produção & 9,7 & 2,8 & 8,3 & 23,6 & 34,7 & 20,8 & 100 \\
\hline $\begin{array}{l}\text { Melhoria em aspectos associados às } \\
\text { regulament. e normas do merc. interno }\end{array}$ & 9,7 & 9,7 & 16,7 & 15,3 & 33,3 & 15,3 & 100 \\
\hline $\begin{array}{l}\text { Melhoria em aspectos associados às } \\
\text { regulament. e normas do merc. externo }\end{array}$ & 16,7 & 6,9 & 16,7 & 13,9 & 30,6 & 15,3 & 100 \\
\hline $\begin{array}{l}\text { Melhoria em aspectos associados à } \\
\text { segurança ou saúde }\end{array}$ & 13,9 & 5,6 & 13,9 & 20,8 & 27,8 & 18,1 & 100 \\
\hline Redução do impacto ambiental & 12,5 & 5,6 & 9,7 & 30,6 & 29,2 & 12,5 & 100 \\
\hline
\end{tabular}

A ampliação da gama de produtos ofertados constitui a principal contribuição da atividade de inovação identificada pelas empresas. Na sequiência, foram consideradas importantes as contribuições relativas à melhoria da qualidade dos produtos, ao aumento da capacidade de produção, à melhoria da flexibilidade de produção, à entrada em novos mercados, à ampliação da participação da empresa no mercado e à redução dos custos de produção. Os resultados levam ao entendimento de que a principal preocupação das empresas reside na busca de aumento da participação e da competitividade no mercado.

A melhoria em aspectos associados às regulamentações e às normas do mercado interno e externo, bem como em aspectos associados à segurança ou saúde representam outros benefícios considerados altamente importantes para as empresas. A observação e o atendimento das regulamentações e normas são aspectos que limitam a competitividade das empresas nos cenários nacional e internacional.

A redução do impacto ambiental foi menos valorizada pelas empresas, o que pode significar a existência de pouco preparo e conhecimento das empresas acerca da importância estratégica de minimizar o impacto ambiental da atividade produtiva e tecnológica na competitividade atual e futura das empresas. A dificuldade em mensurar a contribuição desse indicador na atividade de inovação pode ser outro motivo da sua pouca valorização pelas empresas.

\section{Evolução dos Indicadores}

Os dados relacionados à avaliação da evolução dos indicadores do desempenho inovador nos últimos cinco anos são apresentados na Tabela 9. 
Tabela 9: Evolução dos Indicadores

\begin{tabular}{|l|c|c|c|c|c|c|c|}
\hline \multicolumn{1}{|c|}{ Indicadores } & \multicolumn{6}{|c|}{ Evolução nos últimos cinco anos (\%) } \\
\cline { 2 - 8 } & NR & MBaixa & Baixa & Média & Alta & MAlta & Total \\
\hline $\begin{array}{l}\text { Participação de produtos novos no total } \\
\text { de vendas }\end{array}$ & 30,6 & 5,6 & 13,9 & 18,1 & 23,6 & 6,9 & 100 \\
\hline $\begin{array}{l}\text { Número total de técnicos de nível } \\
\text { superior vinculados à empresa }\end{array}$ & 26,4 & 6,9 & 8,3 & 27,8 & 19,4 & 11,1 & 100 \\
\hline $\begin{array}{l}\text { Redução de custos decorrentes de } \\
\text { inovações tecnológicas de processo }\end{array}$ & 37,5 & 2,8 & 16,7 & 20,8 & 16,7 & 5,6 & 100 \\
\hline Número de patentes obtidas no Brasil & 50 & 20,8 & 6,9 & 9,7 & 9,7 & 2,8 & 100 \\
\hline Número de patentes obtidas no exterior & 54,2 & 19,4 & 12,5 & 6,9 & 2,8 & 4,2 & 100 \\
\hline
\end{tabular}

A participação de produtos novos no total de vendas constitui-se no indicador que apresentou maior índice de evolução no período considerado, seguido pelo número total de técnicos de nível superior vinculados à empresa e do percentual de redução de custos decorrentes de inovações tecnológicas de processo. Esses dados sugerem que as empresas possam estar ampliando a sua capacidade de inovação em produtos e em processo. Por outro lado pode indicar também maior facilidade por parte das empresas na avaliação da evolução desses indicadores.

Ao mesmo tempo, a evolução do número de patentes no Brasil e no exterior nos últimos cinco anos foi considerada muito baixa. Esse dado pode significar que, apesar da presença de indícios de ampliação da competitividade, as empresas não investem no registro de patentes, devido a fatores culturais e de legislação.

\section{Indicadores Obtidos pela Empresa em 2005}

Os dados relacionados aos valores atribuídos aos indicadores do desempenho inovador em 2005 são apresentados na Tabela 10.

Tabela 10: Indicadores do Desempenho Inovador

\begin{tabular}{l|c|c|c|c|c|c|c}
\hline \multicolumn{1}{c|}{ Indicadores } & \multicolumn{6}{c}{ Valores em 2005 } \\
\cline { 2 - 8 } & $\begin{array}{c}\text { NR } \\
\%\end{array}$ & Média & Mediana & $\begin{array}{c}\text { Desvio } \\
\text { Padrão }\end{array}$ & $\begin{array}{c}\text { Coeficiente } \\
\text { Variação \% }\end{array}$ & Min & Máx \\
\hline $\begin{array}{l}\text { Participação de produtos novos no } \\
\text { total de vendas }\end{array}$ & 56,9 & $20,03 \%$ & 10 & 24,53 & 81,66 & 0 & 100 \\
\hline $\begin{array}{l}\text { Redução de custos de produção } \\
\text { decorrentes de inov. tecn. de processo }\end{array}$ & 73,6 & $9,01 \%$ & 2 & 14,84 & 60,72 & 0 & 50 \\
\hline $\begin{array}{l}\text { Número de patentes obtidas no Brasil } \\
\text { Número de patentes obtidas no }\end{array}$ & 72,2 & 7,2 & 1 & 15,49 & 65,66 & 0 & 68 \\
\hline $\begin{array}{l}\text { Exterior } \\
\text { Número total de técnicos de nível } \\
\text { superior, vinculados à empresa }\end{array}$ & 61,1 & 73,82 & 18 & 138,46 & 53,32 & 1 & 576 \\
\hline
\end{tabular}

As médias de participação de produtos novos no total de vendas e de redução de custos de produção decorrentes de inovações tecnológicas de processo das empresas participantes da amostra podem ser consideradas elevadas, se comparadas à realidade da indústria nacional, tradicionalmente caracterizada pela presença de baixos índices de inovação em produtos. Esses dados corroboram as informações contidas na Tabela 9, segundo a qual a maioria das empresas pesquisadas possui inovação de produto e de processo, devido a que o estudo está focado em um grupo de empresas com perfil inovativo bastante significativo. É importante destacar, ainda, a convergência com os dados apresentados na Tabela 9, que apontam a evolução do percentual de participação de produtos novos no total de vendas e de redução de custos de produção decorrentes de inovações tecnológicas de processo, indicando uma tendência de ampliação da capacidade de inovação e da competitividade das empresas. 
Os valores incipientes apresentados em relação ao número de patentes no Brasil e no exterior não são proporcionais à ampliação da participação de produtos novos, anteriormente verificada. Era de esperar que, ao aumentar o número de produtos novos, fosse ampliado o número de patentes registradas. A explicação para esse fato pode estar na pouca tradição das empresas brasileiras em efetuar o registro das patentes.

A média elevada de técnicos de nível superior está relacionada ao porte superior da maioria das empresas pesquisadas, assim como à natureza das atividades tecnológicas, as quais implicam uma elevação no nível técnico e educacional dos seus colaboradores. O significativo índice de não respostas apresentado verificou-se em razão da dificuldade de apropriação dos valores em alguns casos, especialmente no caso da estimativa de redução de custos de produção, bem como por questões de sigilo empresarial.

\section{CONCLUSÕES}

A caracterização da gestão de fontes externas de informação tecnológica evidenciou que as empresas se utilizam de publicações técnico-científicas e da participação em feiras e exibições na busca de acesso à informação tecnológica. A parceria com outras empresas e universidades são as principais modalidades de acesso às tecnologias adotadas, visando à obtenção de um nível tecnológico superior. A oportunidade de acesso constitui o principal critério que orienta a escolha das fontes externas de informação tecnológica, o que salienta a importância do estabelecimento de uma estrutura de network superior para captação de fontes externas de informação tecnológica. Os principais limitadores do processo de contratação de fontes externas de informação tecnológica são a falta de estrutura e de preparo para gerenciar as implicações decorrentes da atividade de cooperação e dos condicionantes legais e culturais envolvidos. Os principais aspectos que facilitam o relacionamento com os parceiros na gestão de projetos são a existência de relacionamento anterior e a expertise; os principais aspectos que dificultam o relacionamento com os parceiros são a estrutura organizacional e de pessoal e o ritmo de trabalho diferenciado entre os parceiros, confirmando os pressupostos teóricos que apontam o fato de que parcerias bem sucedidas compartilham objetivos, estrutura e conhecimento. O desenvolvimento e a absorção de tecnologia mais eficaz, a melhoria do potencial tecnológico, o acesso a recursos humanos qualificados, o uso compartilhado das instalações de pesquisa e informações tecnológicas são os principais benefícios da parceria e da colaboração na atividade de inovação.

Os principais indicadores de inovação utilizados pelas empresas estão relacionados a produto e/ou processo. No que se refere às contribuições da atividade de inovação, destaca-se a ampliação da gama de produtos ofertados e da ampliação da participação no mercado, a melhoria da flexibilidade de produção, o aumento da capacidade de produção, a melhoria em aspectos associados à segurança ou saúde e a regulamentação e normas do mercado interno. Os indicadores de participação de produtos novos no total de vendas, de redução de custos decorrentes de inovações tecnológicas de processo e do número total de técnicos de nível superior vinculados à empresa apresentaram maior evolução nos últimos cinco anos; os indicadores que revelaram maiores índices no ano de 2005 foram a participação de produtos novos no total de vendas e a redução de custos de produção decorrentes de inovação em processo, evidenciando o aumento na competitividade das empresas pesquisadas.

Com base nas análises efetuadas, pode-se concluir, confirmando os pressupostos teóricos subjacentes, que as empresas brasileiras, de modo geral, ainda não possuem formas de gestão de fontes externas de informação tecnológica sistematizadas que potenciem o desempenho dos esforços inovativos, assim como carecem de mecanismos capazes de mensurar os resultados da atividade tecnológica. Apesar das características inovadoras e das práticas de gestão de fontes externas de informação, não foi possível identificar a presença de uma estratégia explícita de gestão de fontes externas de informação para a inovação nos casos analisados.

Artigo recebido em 22.07.2007. Aprovado em 23.04.2008. 


\section{REFERÊNCIAS BIBLIOGRÁFICAS}

Babbie, E. (1999). Métodos de pesquisa de survey. Belo Horizonte: Editora da UFMG.

Beltramo, J., Mason, G, \& Paul, J. (2004). External knowledge sourcing in different national settings: a comparison of electronics establishments in Britain and France. Research Policy, 33(1), 53-72.

Costa, V. M. G., \& Cunha, J. C. (2001). A universidade e a capacitação tecnológica das empresas. Revista de Administração Contemporânea, 5(1), 61-81.

Eurostat. (2004). Third community innovation survey (CIS 3). Luxemburgo, Community Innovation Statistics, Statistical Office of the European Communities, Eurostat. Recuperado em 27 março, 2005, de http://www.epp.eurostat.ec.europa.eu

Fleury, A., \& Fleury, M. T. (1997). Aprendizagem e inovação organizacional: as experiências de Japão, Coréia e Brasil. São Paulo: Atlas.

Hartman, E. A., Tower, C. B., \& Sebora, T. C. (1994). Information sources and their relationship to organizational innovation in small business. Journal of Small Business Management, 32(1), 3648 .

Laursen, K., \& Salter, A. (2004). Searching high and low: what types of firms use universities as a source of innovation? Research Policy, 33(8), 1201-1215.

Leonard-Barton, D. (1995). Wellsprings of knowledge: building and sustaining the sources of innovation. Boston: Harvard Business School Press.

Linder, J. C., Jarvenpaa, S. L., \& Davenport, T. H. (2003a, March 10). Innovation sourcing strategy matters. Accenture institute for strategic change (pp. 1-16). Recuperado em 03 julho 2007, de http://www.accenture.com

Linder, J. C., Jarvenpaa, S. L., \& Davenport, T. H. (2003b). Toward an innovation sourcing strategy. MIT Sloan Management Review, 44(4), 43-52.

Pace, E. S. U., Basso, L. F. C., \& Silva, M. A. (2003). Indicadores de desempenho como direcionadores de valor. Revista de Administração Contemporânea, 7(1), 37-65.

Porto, G. S., Prado, F. O., \& Plonski, G. A. (2003, Octubre). As fontes de tecnologia no setor de telecomunicações e os fatores motivadores da cooperação. Proceedings of the Seminario LatinoIberoamericano de Gestión Tecnológica, México, DF, México, 10.

Ribault, J. M., Martinet, B., \& Lebidois, D. A. (1995). Gestão de tecnologias. Lisboa: Publicações Dom Quixote.

Salter, A., \& Gann, D. (2002). Sources of ideas for innovation in engineering design. Science and Technology Policy Research, 32(8), 1309-1324.

Sbragia, R, Kruglianskas, I., \& Andreassi, T. (1998, novembro). Os indicadores de P\&D\&E nas empresas mais e menos inovadoras. Anais do Simpósio de Gestão da Inovação Tecnológica, São Paulo, SP, Brasil, 20. 